\title{
Circulating Vimentin Over-Expression in Patients with Oral Sub Mucosal Fibrosis and Oral Squamous Cell Carcinoma
}

\author{
Sadhna Aggarwal ${ }^{1} \cdot$ Baldeep Singh $^{1} \cdot$ Suresh C. Sharma $^{1} \cdot$ Satya N. Das $^{1,2}(0)$
}

Received: 25 October 2021 / Accepted: 28 November 2021 / Published online: 11 January 2022

(C) Association of Otolaryngologists of India 2021

\begin{abstract}
Oral squamous cell carcinoma (OSCC) is one of the most common (90\%) types of oral carcinomas in the world. It is the 2nd most common and 3rd deadliest cancer in India. The lack of early detection marker is one of the major causes of worst prognosis. The vimentin belongs to intermediate filament family proteins which plays significant role in maintaining cellular integrity. Over-expression of vimentin has been widely reported in many epithelial cancers however the information regarding its prevalence in the oral cancers still needs further scientific intervention. The expression level of circulating vimentin protein in serum samples $(n=30)$ of oral submucous fibrosis (OSMF), OSCC patients and healthy controls were measured by performing ELISA. The serum level of vimentin was significantly higher in OSMF $(p<0.01)$ and OSCC $(p<0.003)$ patients as compared to healthy subjects. The circulating vimentin levels showed a gradual increase with increasing disease status (normal $<$ OSMF $<$ OSCC). Circulatory levels of vimentin may ba useful indicator of
\end{abstract}

Satya N. Das

satyandas@gmail.com; satyandas@hotmail.com

Sadhna Aggarwal

micro9sadhna@gmail.com

Baldeep Singh

baldeepsingh@live.com

Suresh C. Sharma

suresh6sharma@yahoo.com

1 Department of Biotechnology and Otorhinolaryngology, All India Institute of Medical Sciences, Ansari Nagar, New Delhi 110029, India

2 Present Address: Emeritus Scientist, Indian Council of Medical Research, Ansari Nagar, New Delhi 110029, India disease progression and as a suitable target for therapeutic intervention of oral submucous fibrosis and oral carcinoma.

Keywords Vimentin - ELISA - Biomarker - OSMF . OSCC

\section{Introduction}

Oral cancer is the neoplasm that includes carcinomas of lip, oral cavity and oropharynx. Oral squamous cell carcinoma (OSCC) is the most common (90\%) type of oral carcinomas. It represents 16 th position for both prevalence and morbidity among all cancers globally. Asia alone shares $74 \%$ burden of worldwide deaths due to oral cancer. It is the 2nd most common and 3rd deadliest cancer in Indians. It is first most prevalent in males and fourth most prevalent cancer types in females in India [1]. Differences in prevalence and morality may be due to different life style and eating habits. Different reports had shown tobacco and alcohol consumption promoting the oral cancer risk leading to the poor survival of oral cancer patients [2,3]. Although there have been advances in chemotherapy and surgery in recent years yet 5 -year survival rate of oral cancer has been low $(50-55 \%)$ from decades [4]. Further early stage tumour are difficult to diagnose because it remain un-noticed until its progression into late stages [5]. Therefore, identification of new plausible biomarkers for early detection is need of the hour. Recently, our lab has established vascular endothelial growth factor (VEFG), galectin-1 and galectin3 etc. as plausible biomarkers for OSCC [6, 7].

Oral submucous fibrosis (OSMF) is collagen related chronic, insidious and premelignant disease of oral cavity and pharynx. It is characterized by inflammation and fibrosis of the submucosal tissues, which sometimes leads 
to dysphagia [8]. The prevalence of OSMF in India has been estimated to range from 0.2 to $2.3 \%$ in males and $1.2-4.6 \%$ in females, with a broad age range from 11 to 60 years. Malignant transformation rate of $2.3-7.6 \%$ is also reported [8]. Chewing betel quid commonly known as gutka increases the risk of OSMF. Study showed higher expression of Ki67, c-Met and IMP3 in OSMF and decreased expression of $\beta$-catenin and analzed their prognostic significane of malignant transformation in OSMF [9]. However, increasing incidence of OSMF need more prognostic markers for its early detection and prevent further malignant transformation.

Vimentin is a cytoskeletal type III intermediate filaments (IFs) protein encoded by single copy gene on chromosome 10p13. It provides integrity and flexibility to cell which ultimately helps in movement and protection [10]. Its expression has been seen in wide variety of cells such as connective tissue, mesenchymal cells, pancreatic precursor cells, sertoli cells, neuronal precursor cells, trophoblast giant cells, fibroblasts, endothelial cells liningblood vessels, renal tubular cells, macrophages, neutrophils, mesangial cells, leukocytes, and renal stromal cells [11]. Monomer of vimentin is composed of conserved $\alpha$-helical (rod) domain flanked by non $\alpha$-helical $N$-terminal (head) and C-terminal (tail) domain. These monomers associates with each other in parallel fashion to constitute coiled coil structure which are building blocks of IFs.

Vimentin expression has been observed in cytoplasm, extra cellularly, cell surface as well as in nucleus. Cytoplasmic vimentin plays important role in cell movement, stress resistance and organelle distribution within the cell. Nuclear vimentin regulate transcription of various genes. Cell surface vimentin possibly play role in cell adhesion [12]. Recent reports have shown the significance of secretory vimentin in coronary artery disease and generating immune responses [13]. It has been shown expression of secretory vimentin by activated macrophages and established its role in enhancing bactericidal activity of macrophages by inducing hydrogen peroxide and superoxide [13]. The role of vimentin in modulating apoptosis in immune cells has also been established in various studies. During the immune responses vimentin functions as homing molecule and direct immune cells toward specific sites [14]. Further, vimentin is also associated with developmental [15], renal [16] and wound healing [17] processes. Recent study has shown the importance of vimentin in binding to SARS-CoV-2 spike protein and antibodies that further leads inhibition of in vitro uptake of SARS-CoV-2 virus-like particles. This provides a strategy to decrease the spread of COVID-19 pandemic [18].

Transcription of vimentin gene has been found to be regulated by various transcription factors such as $N F \kappa B$, AP-1, PEA3 and ZBP-89 etc. NFKB and AP-1 are important transcription factors that are activated during various inflammatory processes suggesting the role of vimentin in immune system. ZBP-89 and PEA3 are associated with various other cancers while the exogenous vimentin activates Wnt pathway in tumour cells and modulates its invasive properties [12, 19]. During epithelial mesenchymal transition (EMT), epithelial cells lose its cell polarity, detach from primary tumour site and migrate to another site. In this process of EMT, vimentin has been shown to play important role. Various studies have established vimentin as EMT marker for myeloid leukaemia, breast cancer and cervix cancer. Expression of vimentin has shown to be elevated in various other cancers like prostate, gastrointestinal, breast, lung and central nervous system etc. [11]. Besides its upregulation in various cancers, expression of vimentin is downregulated in macrophages infected with live Mycobacterium tuberculosis [20]. Downregulation of vimentin also plays important role in drug resistance in ovarian cancer [21]. A study has shown the higher expression of cytoplasmic vimentin in OSMF [22], however significance of soluble circulating vimentin in OSCC and OSMF is yet unknown and needs further investigations.

In this present study, we have compared the expression of circulating vimentin protein through ELISA in serum samples from OSCC, OSMF and normal controls. Further, we correlated the expression levels of vimentin in OSCC and OSMF with the clinico-pathological features of these patients.

\section{Material and Method}

\section{Blood Samples}

Peripheral blood samples were obtained from 30 healthy controls and biopsy proven oral cancer patients (OMSF and OSCC) that attended the BRA-Institute Rotary Cancer Hospital of the All India Institute of Medical Sciences (AIIMS), New Delhi, India. None of the cancer patients received anti-cancer therapy before sampling. Individuals with recent surgery, trauma, pregnancy and / or chronic illness were excluded. Similarly, healthy controls who had got fever or viral infection in the past one week, pregnancy, recent accident or trauma were also excluded from the study. The tumour, clinical and histopathology staging of OSCC and OSMF patients was done as previously described [23]. Subsequently, an institution approved written informed consent was obtained from each participant after clear explanation about objectives and implications of the study. Also, the study protocol was approved by the institute's Ethics Committee. 


\section{Isolation of Serum from Blood}

The blood sample $(5 \mathrm{ml})$ was aseptically drawn from each individual by venipuncture. Serum was separated after coagulation at $4{ }^{\circ} \mathrm{C}$ overnight by centrifuging at $3000 \mathrm{rpm}$ for $20 \mathrm{~min}$, and stored at $-80^{\circ} \mathrm{C}$ in aliquots until further use.

\section{Evaluation of Vimentin Expression in Serum Samples by ELISA}

Circulating level of vimentin was determined by ELISA using commercially available Human Vimentin (VIM) ELISA kit (YH Bioresearch Laboratory, Shanghai, China; catalogue number-YHB3192Hu) as per manufacturer's instructions. All serum samples were estimated in duplicates. The detection range and sensitivity of ELISA kit was $0.2-60 \mathrm{ng} / \mathrm{ml}$ and $0.07 \mathrm{ng} / \mathrm{ml}$ respectively. Lastly, the absorbance was measured at $450 \mathrm{~nm}$ using ELISA Reader, Mini protean tetra system (Bio-Rad, CA, USA).

\section{Statistical Analysis}

The statistical analysis was performed using non-parametric Mann-Whitney U test (two-tailed) to compare between two groups. All statistical analyses were performed using GraphPad PRISM Version 6.0 Software, (La Jolla, CA, USA). P value less than 0.05 was considered to be statistically significant.

\section{Results}

\section{Clinical Profile of Oral Cancer Patients}

The clinicopahtological parameters of the patients have been shown in Table 1. The age of the OSMF and OSCC patients ranged as 28-60 and 21-60 years respectively. Majority of the OMSF (80\%) and OSCC (67\%) patients were male. The majority of OSCC patients $(24 ; 80 \%)$ presented with late stage (III \& IV), however, only 06 patients $(20 \%)$ were with early stage (I \& II) tumours. Tongue $(46.6 \%)$ was the most commonly affected site followed by buccal mucosa (23.3\%) followed by gingivobuccal-sulcus (16.6\%), RMT (6.66\%). The alveolus and lip were the least affected site. Mostly, the OSCC patients (56.66) under study were poorly differentiated. However, the percentages of moderately $(23.3 \%)$ and well differentiated $(20 \%)$ patients were almost similar.

Further, the maximum numbers of the OSMF patients were presented in stage III $(73.3 \%)$ followed by stage IV (16.66\%) and Stage II (10\%). In 14 (46.66) patients, the mouth opening ranged between 25 and $35 \mathrm{~mm}$ and was $\geq 35 \mathrm{~mm}$ in $16(53.3 \%)$ patients.

\section{Serum Level of Vimentin in Oral Cancer Patients}

As shown in Fig. 1, the serum levels of vimentin was significantly higher in OSMF $(40.56 \pm 3.271 \mathrm{ng} / \mathrm{ml}$; $p<0.01)$ and OSCC $(42.99 \pm 3.889 \mathrm{ng} / \mathrm{ml} ; p<0.003)$ patients as compared to healthy subjects $(30.38 \pm 1.379 \mathrm{ng} / \mathrm{ml})$. However, the mean concentration of vimentin in peripheral blood of OSCC patients tends to be higher than OSMF suggesting an increasing trend of vimentin with progression of the disease in the order of normal $<$ benign $<$ malignant conditions.

\section{Discussion}

There are six major classes of IFs which are restricted to specific cell types. Type I and type II are acidic and basic keratins respectively and are expressed in epithelial cells. Type III includes vimentins and desmins found in mesenchymal and muscle cells respectively. Type IV includes neurofilaments (in neurons), type V lamins (in cell nuclei) and type VI type nestin (in embryonic neurons). IF-associated proteins such as plectin, ankyrin, desmoplakin, and filaggrin generally link IFs to subcellular components. Vimentin $(57 \mathrm{kDa})$ is a highly conserved protein that belongs to type III IFs family proteins [11]. Vimentin assembles with itself and other proteins (type III and Type IV family proteins) to form homopolymer and heteropolymers in a coiled coil form [24]. Being a cytoskeletal protein, principle function of vimentin is maintaining the integrity and migration. Vimentin has been spotted as cytoskeletal, extracellular as well as nuclear protein [11]. There are several studies which showed the phosphatases and protein kinases play crucial role in assembly and disassembly of vimentin filaments. For example, the phosphorylation of vimentin on S38 and S72 by protein kinase A induces disassembly and dephosphorylation of vimentin by phosphatase $2 \mathrm{~A}$ prevents disassembly $[25,26]$. This repeated process of assembly and disassembly perhaps have crucial role during mitosis, migration as well as protection. Further, various knockout (-/-) studies in mice have shown the importance of vimentin in wound healing, renal function and lymphocyte migration [14, 16, 17]. Recent study has shown the vimentin inhibiting the autophagy wherein, Biskou et al. have shown that vimentin inhibition with WFA accumulates the LC II and autophagosomes which ultimately leads to autophagy [12]. Elevated expression of vimentin has been found in epithelial cells that are undergoing EMT 
Table 1 Clinico-pathological parameters of study subjects

\begin{tabular}{|c|c|c|c|}
\hline Characteristics & OSCC patients & OSMF patients & Controls \\
\hline Age (year) & $28-60$ & $21-60$ & $23-55$ \\
\hline Mean & 45.2 & 35.6 & 32.8 \\
\hline \multicolumn{4}{|l|}{ Gender } \\
\hline Male & $27(90.0)$ & $24(80.0)$ & $20(66.6)$ \\
\hline Female & $03(10.0)$ & $06(20.0)$ & $10(33.3)$ \\
\hline \multicolumn{4}{|l|}{ Site of the tumor } \\
\hline Tongue & $14(46.6)$ & & \\
\hline Buccal mucosa & $07(23.3)$ & & \\
\hline Gingivo-buccal sulcus & 05 (16.6) & & \\
\hline RMT & $02(06.6)$ & & \\
\hline Alveolus & $01(03.3)$ & & \\
\hline Lip & $01(03.3)$ & & \\
\hline \multicolumn{4}{|l|}{ Stage of the tumor } \\
\hline $\mathrm{T} 1+\mathrm{T} 2$ & $06(20.0)$ & & \\
\hline $\mathrm{T} 3+\mathrm{T} 4$ & $24(80.0)$ & & \\
\hline \multicolumn{4}{|l|}{ Lymph node metastasis } \\
\hline No & $10(33.3)$ & & \\
\hline $\mathrm{N}+$ & $20(66.6)$ & & \\
\hline \multicolumn{4}{|l|}{ Histological stage } \\
\hline WDSCC & $06(20.0)$ & & \\
\hline MDSCC & $07(23.3)$ & & \\
\hline PDSCC & $17(56.6)$ & & \\
\hline \multicolumn{4}{|l|}{ OSMF clinical stage } \\
\hline $\mathrm{S} 2$ & & $03(10.0)$ & \\
\hline $\mathrm{S} 3$ & & $22(73.3)$ & \\
\hline $\mathrm{S} 4$ & & $05(16.6)$ & \\
\hline \multicolumn{4}{|l|}{ Mouth opening } \\
\hline M1 & & $16(53.3)$ & \\
\hline $\mathrm{M} 2$ & & $14(46.6)$ & \\
\hline \multicolumn{4}{|l|}{ Histological grading } \\
\hline Very early & & $06(20.0)$ & \\
\hline Early & & $07(23.3)$ & \\
\hline Moderately advanced & & $14(46.6)$ & \\
\hline Advanced & & $03(10.0)$ & \\
\hline
\end{tabular}

transitions. However, during the reverse EMT its expression declines, suggesting its significance in EMT [27].

Extracellular vimentin exists in three forms i.e. "membrane bound", "vesicle coated" and "soluble". Vimentin can be exposed to surface or secreted by several cell types like macrophages, neutrophils, endothelial cells and platelets. Various post translational modifications of vimentin like phosphorylation, tyrosylation and glutathionylation act as signals for its extracellular transport [28]. It has been found that membrane bound vimentin can interact with pathogenic proteins and help immune system to eliminate the them [29]. Another study has shown vimentin expressed on cell surface of platelets bound with Von Willebrandt Factor (VWF) at the site of injury through the tail domain [30]. Vesicle coated vimentin acts as vesicle transported protein and transports vimentin from one site to another [28]. Soluble vimentin can bind antagonistically to P-Selectin on endothelial cells blocking its binding to Pselectin glycoprotein ligand on leukocyte resulting in attenuation of inflammation [31]. Soluble Vimentin can also bind with the CD44 and Dectin on various cell types and modulate cellular function [28].

Risk of OSCC and OSMF is increasing continuously in whole world and specially in India where oral cancer is the 


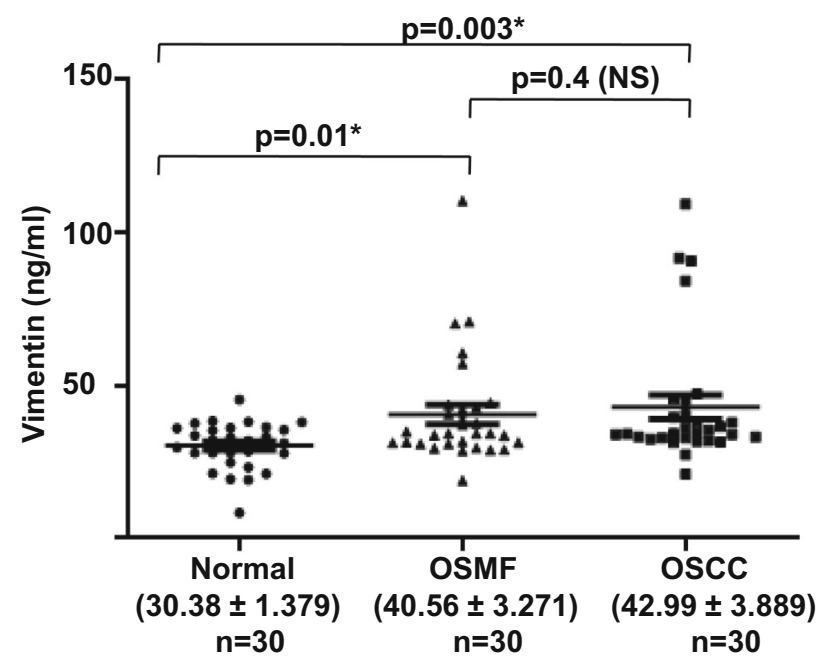

Fig. 1 Circulating levels of vimentin protein in oral cancer. The difference between the variables was measured using Mann-Whitney $\mathrm{U}$ test. The numerical values under $\mathrm{x}$-axis and above each variable represent mean \pm SEM $(\mathrm{ng} / \mathrm{ml})$ and $p$ values respectively. The $p$ values $<0.05$ were considered as statistically significant. Note: $[\mathrm{n}=$ no. of subjects involved, $* p$ value significant, NS (nonsignificant)]

second most common cancer. Hence, the new strategies for detecting and curing OSCC and OSMF are needed. Recently, our lab has found that disrupting the PI3K/Akt/ mTOR signalling through PI3K inhibitors leads to inhibition of tumour growth in OSCC [32]. Several reports have shown the significance of cytoplasmic vimentin as prognostic marker in OSCC [33]. However, the significance of soluble form of vimentin in OSCC and OSMF needed more investigation. Here, we have analysed the serum levels of the vimentin (circulating as soluble form) in OSCC, OSMF patients and compared these with normal controls. We found that the circulating vimentin level was significantly higher in OSMF $(40.56 \pm 3.271 \mathrm{ng} / \mathrm{ml} ; 0.01)$ and OSCC $(42.99 \pm 3.889 \mathrm{ng} / \mathrm{ml} ; 0.003)$ patients as compared to healthy subjects $(30.38 \pm 1.379 \mathrm{ng} / \mathrm{ml})$. However, the mean concentration of vimentin in peripheral blood of OSCC patients was higher than OSMF patients but the difference was statistically insignificant $(p=0.4)$. High level of serum vimentin may act as immune evasive mechanism in OSCC as it attenuates the inflammation as described earlier [31]. Higher levels of soluble vimentin may also enhance invasive properties of cancer cells in OSCC patients [19]. Further, the role of serum vimentin and its prognostic significance in OSCC and OSMF patients could also be investigated. We also analysed the clinico-pathological features of study subjects and found most of the OSCC (67\%) and OSMF (80\%) patients were male, representing late stage. In most of the OSCC patients, site of tumour was tongue $(46.6 \%)$ followed by buccal mucosa $(23.3 \%)$ and gingivo-buccal-sulcus
$(16.6 \%)$. In severe cases of OSMF, patients face difficulties in opening the mouth which leads to further problems in breathing and eating. In this study, $46.6 \%$ patients whose mouth opening ranged between 25 and $35 \mathrm{~mm}$ and patients (53.3\%) whose mouth opening range was $\geq 35 \mathrm{~mm}$ were included. Vimentin is also a drug target in various carcinomas. The drugs such as withaferin-A, silibinin, and salinomycin have been shown to reduce the tumour progression and EMT by decreasing the expression of vimentin. Some micro RNAs like miR-17-3p, miR-200, miR30 have been shown to decrease the vimentin expression and ultimately tumour growth and progression [11]. The new drugs that can either directly bind or inactivate soluble vimentin can be considered as a significant strategy for treating the OSCC and/or OSMF patients.

Here, for the first time in our best knowledge, we have investigated the soluble form of vimentin in blood stream of OSCC and OMSF patients; and shown the elevated levels of circulating vimentin both in OSCC and OSMF patients. Its elevated level in advanced stages may be indicative of tumour growth and progression. The current study surely needs to be concluded further by increasing sample size and also observing the effect of vimentin inhibition on growth of cancer cells in vitro and in vivo. At present we can state that circulatory levels of vimentin could be of prognostic significance and its silencing could be an important strategy for the treatment of OSMF and OSCC.

\section{Declarations}

Conflict of interest No potential conflicts of interest were declared.

Human Participants and/or Animals The research involved Human Participants. The study protocol was approved by 'Ethics Committee' of the All India Institute of Medical Sciences.

Informed Consent Informed consent was obtained from all the participants.

\section{References}

1. IARC Inc(2020): India fact sheet 2020. 361: 2

2. Jerjes W, Upile T, Radhi H et al (2012) The effect of tobacco and alcohol and their reduction/cessation on mortality in oral cancer patients: Short communication. Head Neck Oncol 4:6. https://doi.org/10.1186/1758-3284-4-6

3. Deleyiannis FWB, Thomas DB, Vaughan TL, Davis S (1996) Alcoholism: Independent predictor of survival in patients with head and neck cancer. J Natl Cancer Inst 88:542-549. https://doi.org/10.1093/jnci/88.8.542

4. Silverman S (2001) Demographics and occurrence of oral and pharyngeal cancers: The outcomes, the trends, the challenge. J Am Dent Assoc 132:7S-11S. https://doi.org/10.14219/jada.archive.2001.0382 
5. Khanna NN, Srivastava PK, Khanna S, Das SN (1983) Intensive combination chemotherapy for cancer of the oral cavity. Cancer 52:790-793

6. Aggarwal S, Devaraja K, Sharma SC, Das SN (2014) Expression of vascular endothelial growth factor (VEGF) in patients with oral squamous cell carcinoma and its clinical significance. Clin Chim Acta 436:35-40. https://doi.org/10.1016/j.cca.2014.04.027

7. Aggarwal S, Sharma SC, Das SN (2015) Galectin-1 and galectin3: Plausible tumour markers for oral squamous cell carcinoma and suitable targets for screening high-risk population. Clin Chim Acta 442:13-21. https://doi.org/10.1016/j.cca.2014.12.038

8. Ali FM, Patil A, Patil K, Prasant MC (2014) Oral submucous fibrosis and its dermatological relation. Indian Dermatol Online J 5:260-265. https://doi.org/10.4103/2229-5178.137772

9. Bazarsad S, Zhang X, Kim KY et al (2017) Identification of a combined biomarker for malignant transformation in oral submucous fibrosis. J Oral Pathol Med 46:431-438. https://doi.org/10.1111/jop.12483

10. Messica Y, Laser-Azogui A, Volberg T et al (2017) The role of vimentin in regulating cell invasive migration in dense cultures of breast carcinoma cells. Nano Lett 17:6941-6948. https://doi.org/10.1021/acs.nanolett.7b03358

11. Satelli A, Li S (2011) Vimentin in cancer and its potential as a molecular target for cancer therapy. Cell Mol Life Sci 68:3033-3046. https://doi.org/10.1007/s00018-011-0735-1

12. Ivaska J, Pallari HM, Nevo J, Eriksson JE (2007) Novel functions of vimentin in cell adhesion, migration, and signalling. Exp Cell Res 313(10):2050-2062. https://doi.org/10.1016/j.yexcr.2007.03.040

13. Mor-Vaknin N, Punturieri A, Sitwala K, Markovitz DM (2003) Vimentin is secreted by activated macrophages. Nat Cell Biol 5:59-63. https://doi.org/10.1038/ncb898

14. Nieminen M, Henttinen T, Merinen M et al (2006) Vimentin function in lymphocyte adhesion and transcellular migration. Nat Cell Biol 8:156-162. https://doi.org/10.1038/ncb1355

15. Franke WW, Grund C et al (1982) Formation of cytoskeletal elements during mouse embryogenesis: III. primary mesenchymal cells and the first appearance of vimentin filaments. Differentiation

23:43-59. https://doi.org/10.1111/j.1432-0436.1982.tb01266.x

16. Terzi F, Henrion D, Colucci-Guyon E et al (1997) Reduction of renal mass is lethal in mice lacking vimentin: role of endothelinnitric oxide imbalance. J Clin Invest 100:1520-1528. https://doi.org/10.1172/JCI119675

17. Eckes B, Colucci-Guyon E, Smola H et al (2000) Impaired wound healing in embryonic and adult mice lacking vimentin. J Cell Sci 113(1):2455-2462

18. Suprewicz L, Swoger M, Gupta S et al (2021) Vimentin binds to SARS-CoV-2 spike protein and antibodies targeting extracellular vimentin block in vitro uptake of SARS-CoV-2 virus-like particles. BioRxiv Prepr Serv Biol. https://doi.org/10.1101/2021.01.08.425793

19. Satelli A, Hu J, Xia X, Li S (2016) Potential function of exogenous vimentin on the activation of Wnt signaling pathway in cancer cells. J Cancer 7:1824-1832. https://doi.org/10.7150/jca.15622

20. Mahesh PP, Retnakumar RJ, Mundayoor S (2016) Downregulation of vimentin in macrophages infected with live
Mycobacterium tuberculosis is mediated by reactive oxygen species. Sci Rep 6:1-12. https://doi.org/10.1038/srep21526

21 Huo Y, Zheng Z, Chen Y et al (2016) Downregulation of vimentin expression increased drug resistance in ovarian cancer cells. Oncotarget https://doi.org/10.18632/oncotarget.9970

22. Chang Y-C, Tsai C-H, Tai K-W et al (2002) Elevated vimentin expression in buccal mucosal fibroblasts by arecoline in vitro as a possible pathogenesis for oral submucous fibrosis. Oral Oncol 38:425-430. https://doi.org/10.1016/s1368-8375(01)00083-5

23. Thota R, Aggarwal S, Chirom AS et al (2020) Serum survivin in oral submucosal fibrosis and squamous cell carcinoma Indian. J Otolaryngol Head Neck Surg. https://doi.org/10.1007/s12070-020-01980-0

24. Goldie KN, Wedig T, Mitra K et al (2007) Dissecting the 3-D structure of vimentin intermediate filaments by cryo-electron tomography. J Struct Biol 158:378-385. https://doi.org/10.1016/j.jsb.2006.12.007

25. Eriksson JE, He T, Trejo-Skalli AV et al (2004) Specific in vivo phosphorylation sites determine the assembly dynamics of vimentin intermediate filaments. J Cell Sci 117:919-932. https://doi.org/10.1242/jcs.00906

26. Turowski P, Myles T, Hemmings BA et al (1999) Vimentin dephosphorylation by protein phosphatase $2 \mathrm{~A}$ is modulated by the targeting subunit B55. Mol Biol Cell 10:1997-2015. https://doi.org/10.1091/mbc.10.6.1997

27. Chaffer CL, Brennan JP, Slavin JL et al (2006) Mesenchymal-toepithelial transition facilitates bladder cancer metastasis: role of fibroblast growth factor receptor-2. Cancer Res 66:11271-11278. https://doi.org/10.1158/0008-5472.CAN-06-2044

28. Ramos I, Stamatakis K, Oeste CL, Pérez-Sala D (2020) Vimentin as a multifaceted player and potential therapeutic target in viral infections. Int $\mathrm{J}$ Mol Sci 21:1-27. https://doi.org/10.3390/ijms21134675

29 Stevens HP, Nim ER et al (2013) The intermediate filament protein, vimentin, is a regulator of NOD2 activity. Gut 62:695-707. https://doi.org/10.1136/gutjnl-2011-301775

30. Da Q, Behymer M, Correa JI et al (2014) Platelet adhesion involves a novel interaction between vimentin and von Willebrand factor under high shear stress. Blood 123:2715-2721. https://doi.org/10.1182/blood-2013-10-530428

31. Lam FW, Da Q, Guillory B, Cruz MA (2018) Recombinant human vimentin binds to P-selectin and blocks neutrophil capture and rolling on platelets and endothelium. J Immunol 200:1718-1726. https://doi.org/10.4049/jimmunol.1700784

32. Aggarwal S, John S, Sapra L et al (2019) Targeted disruption of $\mathrm{PI} 3 \mathrm{~K} / \mathrm{Akt} / \mathrm{mTOR}$ signaling pathway, via PI3K inhibitors, promotes growth inhibitory effects in oral cancer cells, Cancer Chemother. Pharmacol 83:451-461. https://doi.org/10.1007/s00280-018-3746-x

33. Liu S, Liu L, Ye W et al (2016) High vimentin expression associated with lymph node metastasis and predicated a poor prognosis in oral squamous cell carcinoma. Sci Rep 6:1-9. https://doi.org/10.1038/srep38834

Publisher's Note Springer Nature remains neutral with regard to jurisdictional claims in published maps and institutional affiliations. 